\title{
Atrial thrombi detection prior to pulmonary vein isolation: Diagnostic accuracy of cardiac computed tomography versus transesophageal echocardiography
}

\author{
Sohaib Munir ${ }^{1}$, Justues H. Chang ${ }^{2}$, Shafeequr R. Salahudeen ${ }^{3}$, Adrian Baranchuk ${ }^{4}$, \\ Cheryl Morris ${ }^{4}$, Micheal O’Reilly ${ }^{4}$, Raveen S. Pal ${ }^{4}$ \\ ${ }^{1}$ Division of Medical Imaging, Western University, London, Ontario, Canada \\ ${ }^{2}$ Division of Orthopedic Surgery, University of Toronto, Toronto, Ontario, Canada \\ ${ }^{3}$ Division of Radiology, Queen's University, Kingston, Ontario, Canada \\ ${ }^{4}$ Division of Cardiology, Queen's University, Kingston, Ontario, Canada
}

\begin{abstract}
Background: Patients routinely undergo transesophageal echocardiography (TEE) prior to pulmonary vein isolation (PVI) in order to rule out the presence of intra-atrial thrombi. Cardiac computed tomography (CCT) is also routinely conducted prior to the procedure to determine cardiac anatomy. Although it has been demonstrated that CCT can also rule out intra-atrial thrombi, the use of CCT for thrombi detection is controversial. The primary objective was to determine the utility of CCT for detection of atrial thrombi as compared to TEE.
\end{abstract}

Methods: Patients who underwent PVI between 2010 and 2011 with CTs and TEEs completed within 3 days of each other were retrospectively identified. TEE reports were analyzed, while CCTs were interpreted by a cardiologist specializing in CCTs. Severe spontaneous echo contrast or thrombus detected on TEE were considered positive, as were filling defects found on CCT.

Results: A total of 51 patients undergoing PVI (mean age $59.4 \pm 9.5$ years; $75 \%$ male; ejection fraction $60 \pm 12 \%$ ) had both TEE and CCT in timely fashion. By TEE, 0 left atrial appendage (LAA) thrombi were identified with mild to moderate spontaneous echo contrast in 4 patients. By CCT, 2 definite LAA thrombi were identified and thrombi in 4 patients could not be ruled out. Specificity, positive predictive value, and negative predictive value for CCT were $88 \%, 0 \%$, and $100 \%$, respectively.

Conclusions: CCT is an effective tool in ruling out atrial thrombi prior to PVI. TEE should be completed only if CCT is positive. (Cardiol J 2015; 22, 5: 576-582)

Key words: computed tomography scan, transesophageal echo, cardiac clot, pulmonary vein isolation

\section{Introduction}

Atrial fibrillation (AF) is a common arrhythmia and carries a considerable degree of morbidity [1].
AF causes a predisposition to thrombosis within the left atrium (LA) and left atrial appendage (LAA) due to blood stasis, and increases the long-term risk of ischemic stroke secondary to a cardioembol-

Address for correspondence: Raveen S. Pal, MD, FRCPC, Division of Cardiology, Queen's University, 76 Stuart St, KGH, FAPC3, Kingston, ON, K7N 3N6, Canada, 613-549-6666 x 6245, 613-548-1387, e-mail: rs.pal@queensu.ca 
ic events [2]. Therefore, patients are administered anticoagulants, depending on their risk of stroke as assessed by the $\mathrm{CHADS}_{2}$ score $[1,2]$.

Current standard of care for patients undergoing pulmonary vein isolation (PVI) [3], a common treatment of AF, involves two imaging tests [4]. Transesophageal echocardiography (TEE) imaging is required to rule out the presence of intracardiac thrombus prior to PVI [5]. Cardiac computed tomography (CCT) imaging is also required for procedural mapping. Although it has been demonstrated that CCT can also effectively rule out intracardiac thrombi [6], studies are small and results have been inconsistent in findings and methodology [5,7-14]. For instance, the largest study on the subject by Martinez et al. [9], reached the conclusion that CCT is sensitive enough to warrant the use of only CCT to rule out atrial thrombi, but this conclusion has been called into question for sub-optimal study methodology [15]. Variations in prospective and retrospective electrocardiogram (ECG-gating), patient positioning, and length of time between CCT and TEE are factors that have been inconsistent across studies. As a result of this controversy, patients continue to undergo both TEE and CCT procedures prior to PVI.

The aim of this study is to determine the diagnostic accuracy of CCT compared to TEE in excluding LA or LAA thrombi prior to PVI.

\section{Methods}

\section{Study population}

Consecutive patients selected for PVI by the Arrhythmia Service of Kingston General Hospital, Ontario, Canada between 2010 and 2011 were included in this study. This included both outpatient and inpatient subjects who underwent both TEE and CCT prior to PVI. Exclusion criteria included any patients who did not have TEE and CCT within 3 days of each other. The study protocol was approved by the Health Sciences Research Ethics Board at Queen's University, Kingston, Ontario.

\section{TEE technical details}

TEE images were acquired in accordance with standard patient preparation, using both GE (E9, Fairfield, Connecticut) and Philips (iE33, Andover, Massachusetts) equipment, by cardiologists experienced in TEE. LA and LAA imaging were performed with 2-dimensional (2D) and occasionally 3D X-plane imaging using a $3.5-7 \mathrm{MHz}$ multiplane probe. All interpreting cardiologists were blinded to patient clinical data. Careful visualization of the LA and LAA in multiple views resulted in a report stating thrombi seen or thrombi not seen. A thrombus was defined as abnormal echogenic material within the intracavitary space. No contrast agents were used to enhance visualization. If present, spontaneous echocardiographic contrast (SEC) was commented on. No comment implied the absence of SEC.

\section{CCT technical details}

CCT was performed with prospective ECG-gating and contrast enhancement with a 64-slice CT scanner, Toshiba (Aquillon One, Irvine, CA). Patients were pretreated with beta-blocker and nitroglycerin spray. CT parameters were set at gantry rotation time of $330 \mathrm{~ms}$, and a tube voltage of $100 \mathrm{kVp}$. Following multiple breath hold exercises, an $80 \mathrm{~mL}$ bolus of iodinated contrast media (Omnipaque 350 [GE Healthcare, Milwaukee, Wisconsin] was injected intravenously) at $5 \mathrm{~mL} / \mathrm{s}$. Bolus tracking was initiated with the administration of $80 \mathrm{cc}$ of contrast. Images were obtained every $2 \mathrm{~s}$ at the level of the descending aorta to monitor for appearance of contrast. Contrast appearance was defined as 140 Houndsfield Units (HU) in the descending aorta. Once contrast was visualized, a breath hold instruction was given and the scan was initiated. Image acquisition was completed with a single breath hold with the patient in the supine position. The entire heart was imaged in one heartbeat. Due to the CT scanner technology of one beat acquisition, delayed scanning and prone imaging, would require a second full acquisition. Therefore, delayed scanning and prone positioning, though they might have increased specificity [16], were not completed in order to limit radiation exposure to the patient.

Reconstructions were performed with a small 180 to $240 \mathrm{~mm}$ field of view centered on the heart with a $0.5 \mathrm{~mm}$ slice thickness. Images were reconstructed into 2D axial, coronal and sagittal slices. Multiplanar reconstruction and 3D volume rendered images were also generated and reviewed when required. No attempt was made to optimize imaging of the LAA. All images available were reviewed by a CCT trained, Level III certified, cardiologist (RSP) who was blind to TEE results, on a Vitrea Origin (Vital Enterprises, Minnetonka, Minnesota) workstation.

\section{Data collection}

Two authors (SM and JC), blind to CCT results, retrieved data from the patient record. Data included age, gender, body surface area, valvular disease, 
prior ischemic disease, type and duration of AF or atrial flutter, and antiplatelet or anticoagulation therapy. Heart rate, blood pressure, creatinine and international normalized ratio at the time of the procedure were also recorded. The presence of contributors to the $\mathrm{CHADS}_{2}$ score was recorded, and the $\mathrm{CHADS}_{2}$ score was calculated. TEE reports were reviewed, from which the absence or presence of LA/LAA thrombus was extracted. Presence or absence of SEC was noted rather than grades of mild, moderate or severe SEC, given that the latter does not have a quantitative definition. Positive thrombi and presence of SEC were both considered true positives by TEE. CCT images were interpreted by a cardiologist trained in CCT (RSP), who was blind to the TEE results. Presence of thrombi was noted as "definite", "cannot rule out", or "absent". "Definite" thrombus by CT was defined as incomplete opacification of the LAA with contrast media. "Cannot rule out" thrombus was defined as incomplete mixing of contrast material in the LAA. "Absent" thrombus was defined as complete opacification of the LAA. Both "definite" and "cannot rule out" findings were considered abnormal CCTs. Radiation doses, HU in the LAA, and physical dimensions of the LA and LAA were also recorded.

\section{Statistical analysis}

Primary outcome measures were thrombi detected by TEE and thrombi detected by CCT. Demographic data was presented as proportions with standard deviations. Quantitative data was presented as means with standard deviations. Sensitivity, specificity, negative predictive value (NPV) and positive predictive value (PPV) were calculated for CCT with TEE results as criterion standard.

\section{Results}

A total of 77 patients underwent PVI preceded by CCT and TEE between 2010 and 2011. Only 51 had both imaging tests, TEE and CCT, within $72 \mathrm{~h}$ of each other, therefore 26 patients were excluded from the analysis. Demographic and risk factor data can be seen in Table 1 . Overall, the 51 subjects had

Table 1. Clinical characteristics of the patients.

\begin{tabular}{|c|c|c|c|}
\hline & $\begin{array}{l}\text { Overall } \\
(n=51)\end{array}$ & $\begin{array}{l}\text { CCT positive } \\
(n=6)\end{array}$ & $\begin{array}{l}\text { Mild to moderate SEC } \\
\qquad(\mathrm{n}=4)\end{array}$ \\
\hline Age [years] & $59.4 \pm 9.5$ & $59.3 \pm 4.8$ & $56.3 \pm 8.8$ \\
\hline Male & $38(75 \%)$ & $6(100 \%)$ & $4(100 \%)$ \\
\hline Body surface area $\left[\mathrm{m}^{2}\right]$ & $2.1 \pm 0.27$ & $2.2 \pm 0.19$ & $2.2 \pm 0.27$ \\
\hline Duration of atrial fibrillation or flutter [years] & $5.0 \pm 6.0$ & $2.5 \pm 1.4$ & $2.8 \pm 1.7$ \\
\hline \multicolumn{4}{|l|}{ Atrial fibrillation: } \\
\hline Persistent & $15(29 \%)$ & $5(83 \%)$ & $2(50 \%)$ \\
\hline Paroxysmal & $39(76 \%)$ & $2(33 \%)$ & $2(50 \%)$ \\
\hline Atrial flutter & $7(14 \%)$ & $1(17 \%)$ & $1(25 \%)$ \\
\hline Anticoagulation therapy & $28(55 \%)$ & $6(100 \%)$ & $3(75 \%)$ \\
\hline Antiplatelet therapy & $14(27 \%)$ & $3(50 \%)$ & $1(25 \%)$ \\
\hline No anticoagulation or antiplatelet therapy & $14(27 \%)$ & $0(0 \%)$ & $1(25 \%)$ \\
\hline International normalized ratio & $1.5 \pm 0.6$ & $2.2 \pm 0.6$ & $1.4 \pm 0.3$ \\
\hline Cardiac heart failure & $2(4 \%)$ & $0(0 \%)$ & $0(0 \%)$ \\
\hline Hypertension & $20(39 \%)$ & $3(50 \%)$ & $0(0 \%)$ \\
\hline Age $\geq 75$ years & $2(4 \%)$ & $0(0 \%)$ & $0(0 \%)$ \\
\hline Diabetes mellitus & $4(8 \%)$ & $0(0 \%)$ & $1(25 \%)$ \\
\hline Prior CVA/TIA & $2(4 \%)$ & $0(0 \%)$ & $0(0 \%)$ \\
\hline \multicolumn{4}{|l|}{$\mathrm{CHADS}_{2}$ score: } \\
\hline 0 & $25(49 \%)$ & $3(50 \%)$ & $3(75 \%)$ \\
\hline 1 & $23(45 \%)$ & $3(50 \%)$ & $1(25 \%)$ \\
\hline 2 & $0(0 \%)$ & $0(0 \%)$ & $0(0 \%)$ \\
\hline 3 & $3(6 \%)$ & $0(0 \%)$ & $0(0 \%)$ \\
\hline
\end{tabular}

CCT — cardiac computed tomography; CVA — cerebro-vascular accident; SEC — spontaneous echocardiography contrast; TIA — transient ischemic attack 
Table 2. Findings by transesophageal echocardiography prior to pulmonary vein isolation.

\begin{tabular}{lc}
\hline LAA thrombus & $0(0 \%)$ \\
Spontaneous echo contrast: & $4(8 \%)$ \\
Mild & 2 \\
Moderate & 2 \\
Severe & 0 \\
Ejection fraction [\%] & $59.5 \pm 12.2$ \\
LA volume [mm ${ }^{2}$ ]: & $31.9 \pm 8.5$ \\
SEC positive (mild, & $37.2 \pm 8.9$ \\
moderate or severe) & \\
SEC negative & $31.4 \pm 8.6$ \\
LA diameter [mm]: & $40.1 \pm 7.2$ \\
SEC positive (mild, & $42.4 \pm 9.4$ \\
moderate or severe) & \\
SEC negative & $39.9 \pm 7.1$ \\
\hline
\end{tabular}

LAA — left atrial appendage; LA — left atrium; SEC — spontaneous echocardiographic contrast

an average age of 59.4 years, $75 \%$ male, $100 \%$ with $\mathrm{AF}$ and $14 \%$ with concomitant atrial flutter, $94 \%$ with $\mathrm{CHADS}_{2}$ score $\leq 1$. CCT positive patients were more likely than TEE positive and overall patients to have persistent $\mathrm{AF}(83 \%, 50 \%$, and $29 \%$, respectively). Patients with definite and possible thrombi by CCT were also more likely to be chronically anticoagulated than TEE positive and overall patients $\left(100 \%, 75 \%, 55 \%\right.$, respectively). $\mathrm{CHADS}_{2}$ scores were not significantly different between the overall subject population and the patients with positive CCT or TEE findings. Anticoagulation and antiplatelet rates represent chronic prophylaxis as all patients received warfarin anticoagulation for 4 weeks prior and following PVI. For TEE, only definite thrombi or severe SEC were considered true positive, since management decisions were not impacted by mild to moderate spontaneous echo contrast. No true thrombi were identified by TEE. No severe SEC was reported on TEE. Mild and moderate SEC was reported in 4 patients in total (Table 2). For CCT, both definite and possible thrombi were considered positive results. Two definite thrombi were identified by CCT. In addition, there were 4 CCT scans in which thrombus could not be ruled out. By TEE, SEC positive patients had larger LA volumes $\left(37.2 \mathrm{~mm}^{2}\right.$ vs. $\left.31.4 \mathrm{~mm}^{2}\right)$, but not greatly different diameters ( $42.4 \mathrm{~mm}$ vs. $39.9 \mathrm{~mm})$. Positive thrombi by CCT also had greater LA volumes compared to negative tests ( $56.3 \mathrm{~mm}$ vs. $42.3 \mathrm{~mm}$ ). It should be noted that $30 \%$ of TEE reports and $8 \%$ of CCT reports did not document LA volumes and thus were not included in our analysis.
Of the 4 patients with mild or moderate SEC by TEE, only 1 had definite or possible thrombi by CCT. Conversely, of the 6 patients with definite or possible thrombi by CCT, none had associated with thrombi or SEC by TEE and were therefore all false positives. Overall, CCT had a specificity of $88 \%$, PPV of $0 \%$, and NPV of $100 \%$. As there were no positive thrombi by TEE, sensitivity for CCT could not be calculated.

\section{Discussion}

Both TEE and CCT have advantages and disadvantages. CCT is rapid and non-invasive. However, it requires contrast administration and radiation exposure to the patient [7]. TEE is accurate and easily accessible. However, it is invasive and is associated with a small risk of esophageal rupture. It also requires sedation due to patient discomfort [17].

Current literature includes both prospective and retrospective cross-sectional studies, and a meta-analysis [5, 7-13, 18]. Study sizes have varied from 72 to 402 patients [7, 9], yet current literature remains controversial. Martinez et al. [9] found sensitivity and specificity of CCT to be $100 \%$ and $92 \%$, respectively concluding that TEE is unnecessary. Similarly, Hur et al. [13] found a sensitivity and specificity of $89 \%$ and $100 \%$. In contrast, Tang et al. [10] conclude from CCT sensitivity and specificity of $36.4 \%$ and $93.7 \%$ that both modalities are necessary. Variation in results can partially be explained by inconsistency in CCT and TEE methodology. Garcia [15] criticized Martinez et al. [9] for suboptimal methodology in administration of contrast, possibly affecting visualization. The clinical impact of prospective and retrospective ECG-gating on CCT results has also been called into question [10], as have variations in patient positioning during CCT [19]. Lastly, the length of time between CCT and TEE has varied, as Martine $z$ et al. ensured CCT and TEE were conducted within $24 \mathrm{~h}$, whereas Kim et al. allowed a gap of up to one week $[5,9,13]$.

The results of this study demonstrate that in a population of patients with AF, CCT has high specificity for detecting absence of LAA thrombus (Fig. 1). Furthermore, CCT has 100\% NPV. However, when CCT is positive, thrombus is at times not visualized on TEE, suggesting that it cannot be used to predict the presence of thrombus. Our findings do not support the routine use of both CCT and TEE prior to PVI. Rather, they suggest that CCT is ideal as the first and only test in patients 


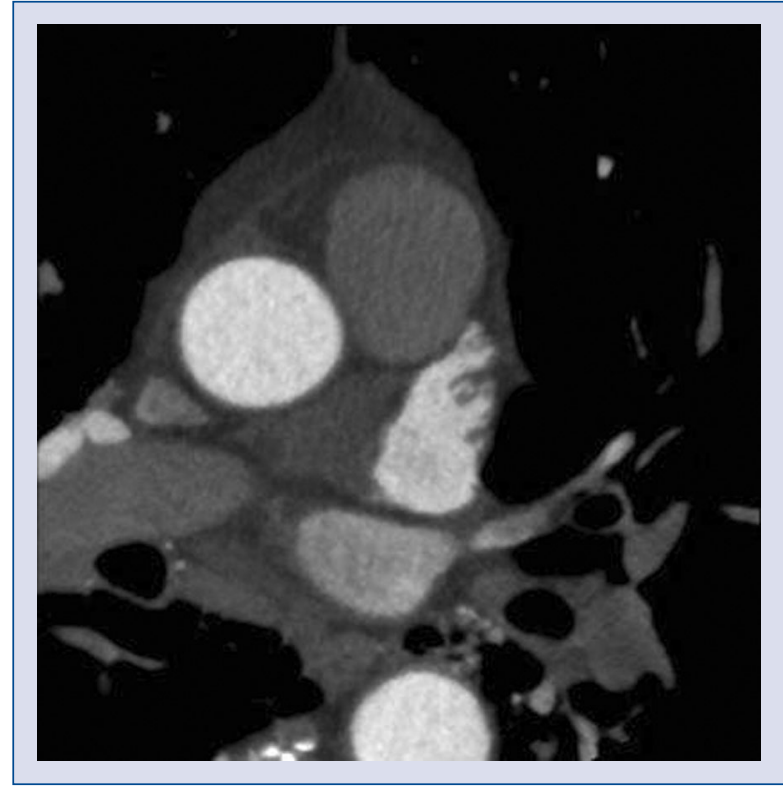

Figure 1. Normal cardiac computed tomography with no left atrial appendage clots.

prior to PVI, with TEE reserved for CCT positive patients. Reduction from two imaging modalities to one would result in significant cost reductions as well as having positive implications for patient safety and comfort. Given the occurrence of false positives in CCT, TEE will still need to be employed to rule out intracardiac thrombi in all cases with possible thrombi by CCT.
The presence of thrombi in patients undergoing PVI is low, reported as $1.2 \%$ by Michael et al. [20]. A more recent study by Hong et al. [21] demonstrated that periprocedural incidence of LAA thrombus identification and stroke is low, further supporting that patients can be screened by CCT alone. None of the patients in our study undergoing PVI was found to have a definite thrombus identified by TEE. This may be related to the low $\mathrm{CHADS}_{2}$ score of the majority of this study population (94\%). However, given the low incidence of thrombus formation in the overall PVI patient population, these findings could be generalizable to higher $\mathrm{CHADS}_{2}$ risk patients as well. Notably, all SEC and CCT positive results did not have a $\mathrm{CHADS}_{2}$ scores $\geq 2$.

Mild to moderate SEC on TEE was not a predictor of a false positive CCT. Only 1 of 4 patients with mild to moderate SEC on TEE had a false positive result on CCT. Also, the other 5 false positive CCT findings did not correspond to any SEC on TEE. Hence, false positive results on CCT rarely corresponded to mild to moderate SEC on TEE. The 2 cases reported as "definite" thrombus on CCT, but were negative on TEE, had unique structural characteristics. One patient had a dihedral LAA, with one head demonstrating decreased contrast density. This may have been due to incomplete mixing of contrast in CCT or due to incomplete visualization of both heads by TEE (Fig. 2A). The other patient had a severely enlarged LA and

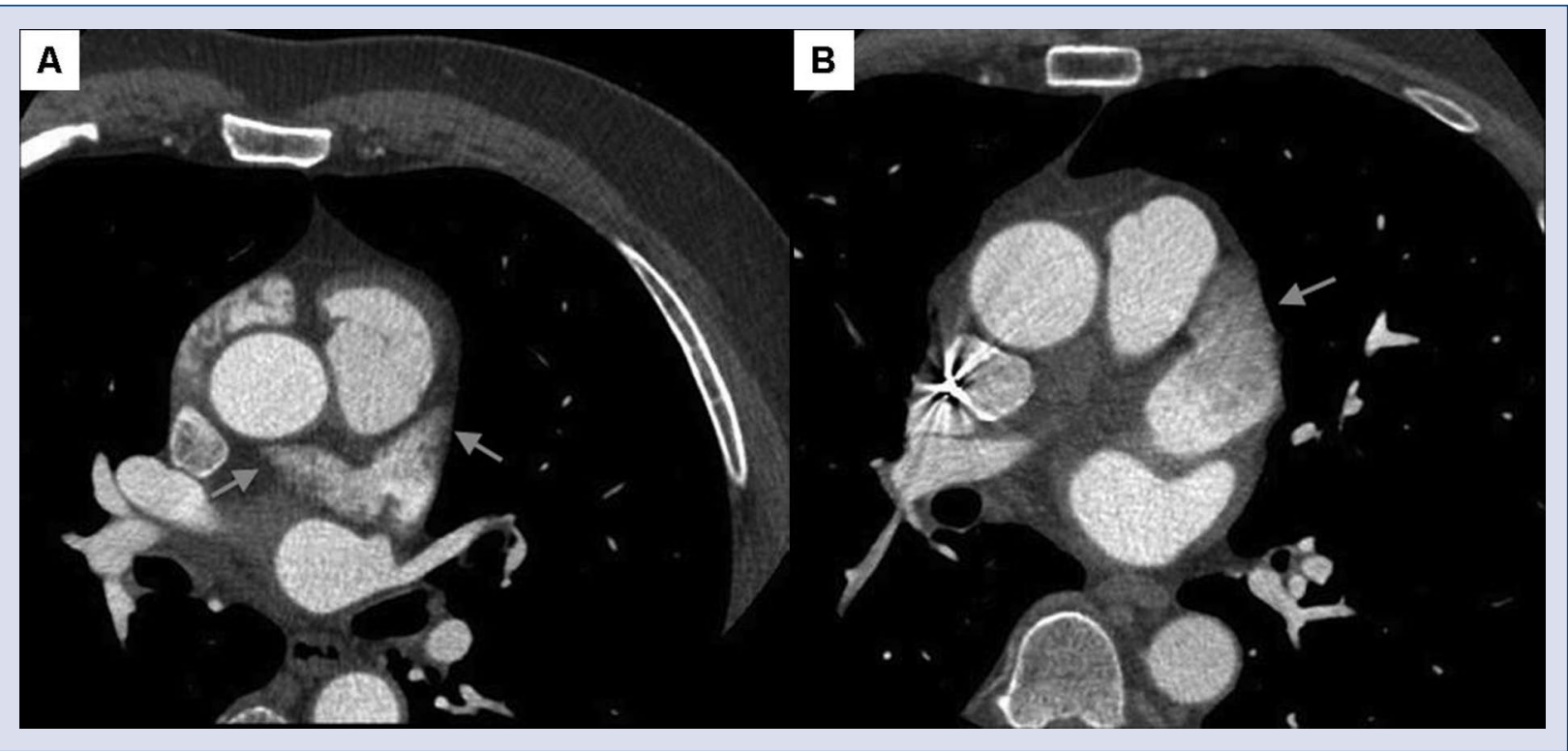

Figure 2. A. Incomplete opacification of the left atrial appendage (LAA) due to a possible dihedral structure of the LAA (gray arrows); B. Another cardiac computed tomography identifying a "definite" thrombus within the LAA (gray arrow), but negative by transesophageal echocardiography. The LAA is severely enlarged leading to incomplete opacification. 
LAA. The LAA had decreasing contrast density in the more distal aspect of the appendage. Again, this may have been secondary to incomplete contrast mixing on CCT or incomplete visualization by TEE (Fig. 2B). Interestingly, both SEC positive and CCT positive tests were associated with larger LA volumes compared to the normal tests. These positive results were likely due to the ineffective contractions secondary to the large LA volumes. These results also suggest that LA size may be the most optimal way to define patients requiring both tests.

Our results are in agreement with Martinez et al. [9], the largest study in this subject area, who reported a specificity of $92 \%$ and a NPV of $100 \%$. This study also drew the conclusion that TEE may be eliminated from routine practice. We hypothesize that our results are similar to Martinez et al. [9] because it is the most recent study, reflecting the evolution of CCT technology and technique over time. With increasing standardization of technology, we anticipate our results to be reproducible.

Similarly, the meta-analysis by Romero et al. [18], which included 19 studies for a total of 2,955 patients, demonstrated a sensitivity and specificity of $96 \%$ and $92 \%$. The PPV and NPV of CCT were $41 \%$ and $99 \%$, respectively. Performing a sub-analysis by limiting the studies to those with delayed imaging increased the sensitivity and specificity to $100 \%$ and $99 \%$, respectively, yielding a PPV and NPV of $92 \%$ and $100 \%$. However, the study by Romero et al. [18] suffered from varying results in assessment of publication bias, based on the test used. Finally, a large study by Hong et al. [21] further supports the low incidence of both LAA thrombus and stroke periprocedural to PVI.

\section{Limitations of the study}

Our study is limited by its small sample size, which when compounded with a low rate of atrial thrombi by CCT or TEE, prevents detailed statistical analysis. Delayed scanning, which increases specificity of CCT by decreasing detection of pseudothrombi, was not routinely completed at our center.

\section{Conclusions}

CCT presents high specificity and NPV for detection of atrial thrombi. TEE should be used to rule out thrombi only in patients with positive CCT results or severely enlarged LA size.
Founding: Part of this study was funded by the Mach-Gaensslen Foundation of Canada, Ottawa, Ontario, Canada.

\section{Conflict of interest: None declared}

\section{References}

1. Wyse DG, Waldo AL, DiMarco JP et al. A comparison of rate control and rhythm control in patients with atrial fibrillation. N Engl J Med, 2002; 347: 1825-1833.

2. Kim D, Chung JW, Kim CK et al. Impact of CHADS(2) score on neurological severity and long-term outcome in atrial fibrillation-related ischemic stroke. J Clin Neurol, 2012; 8: 251-258.

3. Kim YH. Catheter ablation of longstanding persistent atrial fibrillation. Are we on the right path? Circ J, 2012; 76: 1299-1306.

4. Beigel R, Wunderlich NC, Ho SY, Arsanjani R, Siegel RJ. The left atrial appendage: anatomy, function, and noninvasive evaluation. J Am Coll Cardiol Cardiovasc Imag, 2014; 7: 1251-1265.

5. Kim YY, Klein AL, Halliburton SS et al. Left atrial appendage filling defects identified by multidetector computed tomography in patients undergoing radiofrequency pulmonary vein antral isolation: A comparison with transesophageal echocardiography. Am Heart J, 2007; 154: 1199-1205.

6. Geller JC, Brunelli M. Catheter ablation of atrial fibrillation without prior transoesophageal echocardiography: Are we there yet? Europace, 2011; 13: 453-454.

7. Patel A, Au E, Donegan K et al. Multidetector row computed tomography for identification of left atrial appendage filling defects in patients undergoing pulmonary vein isolation for treatment of atrial fibrillation: Comparison with transesophageal echocardiography. Heart Rhythm, 2008; 5: 253-260.

8. Hur J, Kim YJ, Lee HJ et al. Dual-enhanced cardiac CT for detection of left atrial appendage thrombus in patients with stroke: A prospective comparison study with transesophageal echocardiography. Stroke, 2011; 42: 2471-2477.

9. Martinez MW, Kirsch J, Williamson EE et al. Utility of nongated multidetector computed tomography for detection of left atrial thrombus in patients undergoing catheter ablation of atrial fibrillation. J Am Coll Cardiol Cardiovasc Imag, 2009; 2: 69-76.

10. Tang RB, Dong JZ, Zhang ZQ et al. Comparison of contrast enhanced 64-slice computed tomography and transesophageal echocardiography in detection of left atrial thrombus in patients with atrial fibrillation. J Interv Card Electrophysiol, 2008; 22: 199-203.

11. Jaber WA, White RD, Kuzmiak SA et al. Comparison of ability to identify left atrial thrombus by three-dimensional tomography versus transesophageal echocardiography in patients with atrial fibrillation. Am J Cardiol, 2004; 93: 486-489.

12. Gottlieb I, Pinheiro A, Brinker JA et al. Diagnostic accuracy of arterial phase 64-slice multidetector CT angiography for left atrial appendage thrombus in patients undergoing atrial fibrillation ablation. J Cardiovasc Electrophysiol, 2008; 19: 247-251.

13. Hur J, Pak HN, Kim YJ et al. Dual-enhancement cardiac computed tomography for assessing left atrial thrombus and pulmonary veins before radiofrequency catheter ablation for atrial fibrillation. Am J Cardiol, 2013; 112: 238-244.

14. Budoff MJ, Shittu A, Hacioglu Y et al. Comparison of transesophageal echocardiography versus computed tomography for 
Cardiology Journal 2015, Vol. 22, No. 5

detection of left atrial appendage filling defect (thrombus). Am J Cardiol, 2014; 113: 173-177.

15. Garcia MJ. Detection of left atrial appendage thrombus by cardiac computed tomography: a word of caution. J Am Coll Cardiol Cardiovasc Imag, 2009; 2: 77-79.

16. Sawit ST, Garcia-Alvarez A, Suri B et al. Usefulness of cardiac computed tomographic delayed contrast enhancement of the left atrial appendage before pulmonary vein ablation. Am J Cardiol, 2012; 109: 677-684.

17. Ramadan AS, Stefanidis C, Ngatchou W, LeMoine O, De Canniere D, Jansens JL. Esophageal stents for iatrogenic esophageal perforations during cardiac surgery. Ann Thorac Surg, 2007; 84: 1034-1036.

18. Romero J, Husain SA, Kelesidis I, Sanz J, Medina HM, Garcia MJ. Detection of left atrial appendage thrombus by cardiac computed tomography in patients with atrial fibrillation: a meta-analysis. Circ Cardiovasc Imag, 2013; 6: 185-194.

19. Tani T, Yamakami S, Matsushita T et al. Usefulness of electron beam tomography in the prone position for detecting atrial thrombi in chronic atrial fibrillation. J Comput Assist Tomogr, 2003; 27: 78-84.

20. Michael KA, Redfearn DP, Baranchuk A et al. Transesophageal echocardiography for the prevention of embolic complications after catheter ablation for atrial fibrillation. J Cardiovasc Electrophysiol, 2009; 20: 1217-1222.

21. Hong SJ, Kim JY, Kim JB et al. Multidetector computed tomography may be an adequate screening test to reduce periprocedural stroke in atrial fibrillation ablation: A multicenter propensity-matched analysis. Heart Rhythm, 2014; 11: 763-770. 